\section{Andreas Unger}

\section{Epidemiologie}

Mit einer Lebenszeitprävalenz von 85\% gehören Rückenschmerzen $\mathrm{zu}$ den am häufigsten angegebenen Schmerzen [1]. Davon Betroffene berichten über eine lange Erkrankungsdauer in Form von multiplen, rekurrierenden Episoden oder chronisch kontinuierlichen Rückenschmerzen. Frauen sind häufiger betroffen als Männer. Rückenschmerzen führen seit Jahren die Statistiken der Anlässe für Arbeitsunfähigkeit und medizinische Rehabilitation an. Damit sind hohe direkte Kosten für Diagnostik und Therapie als auch indirekte Kosten für Arbeitsunfähigkeit und Frühberentung verbunden.

\section{Risikofaktoren für eine Chronifizierung}

Die 2011 in Deutschland von einer Vielzahl von Fachgesellschaften herausgegebene «Nationale VersorgungsLeitlinie Kreuzschmerz» [2] (Kreuzschmerzen werden als Schmerzen im Rückenbereich unterhalb des Rippenbogens, oberhalb der Gesässfalten, mit oder ohne Ausstrahlung definiert) beschreibt mit «red flags» Warnhinweise auf eine spezifische vertebragene Ursache mit oft dringendem Handlungsbedarf - wie Fraktur, Tumor, Infektion und Radikulopathien/Neuropathien.

Etwa 90\% der Fälle haben keine erkennbare Ursache und werden als unspezifische Rückenschmerzen bezeichnet [3]. Mit «yellow flags» werden die Risikofaktoren für eine Chronifizierung des akuten Kreuzschmerzes bezeichnet - wie die psychosozialen Risikofaktoren Depressivität, Dis-

\title{
Integrative Schmerztherapie bei chronischem Rückenschmerz
}

Jedes schulmedizinische sowie komplementärmedizinische Verfahren, wie z.B. Neuraltherapie, Akupunktur/TCM oder Manuelle Therapie/Osteopathie, stösst bei noch so gekonnter Durchführung an Grenzen. Gezielte Kombinationen von Therapien auf struktureller, biomechanischer, myofaszialer und bioenergetischer Ebene, begleitender Psychotherapie, Berücksichtigung von Umweltbelastungen sowie Substitution von Vitaminen und Spurenelementen führen häufig weiter. Die Notwendigkeit von Integrativer Schmerztherapie ergibt sich nicht nur aus dem Wunsch des Patienten nach einer nebenwirkungsarmen Therapie, sondern auch aufgrund der Grenzen klassischer schulmedizinischer Verfahren.

stress (vor allem berufs-/arbeitsbezogen), schmerzbezogene Kognitionen (wie Katastrophisieren, Hilf-/Hoffnungslosigkeit, Angst-Vermeidungsverhalten (Fear-Avoidance Beliefs)) und passives Schmerzverhalten (ausgeprägtes Schon- und Vermeidungsverhalten), berufliche Faktoren wie körperliche Schwerarbeit, monotone Körperhaltung, geringe berufliche Qualifikation, berufliche Unzufriedenheit und iatrogene Faktoren wie übertriebener Einsatz diagnostischer Massnahmen, Überbewertung somatischer/radiologischer Befunde bei nichtspezifischen Schmerzen und mangelhafte Respektierung der multikausalen Genese [2].

Anders als z.B. bei einem in der Magnetresonanztomografie nachgewiesenen akuten Bandscheibenvorfall mit klinisch dazu passender radikulärer Symptomatik, für den ein operativer Eingriff ein sinnvolles therapeutisches Vorgehen sein könnte, versagen beim chronischen Schmerz unimodal auf Ursache und Wirkung ausgerichtete Therapien. Und selbst bei den radikulär bedingten Kreuzschmerzen ist letztlich in der Mehrzahl der Fälle eine konservative Therapie zielführend, sodass operative Interventionen Fällen mit gravierenden neurologischen Störungen vorbehalten bleiben sollten.

\section{Krankheitsmodelle}

Nicht nur der Patient, sondern auch die behandelnden Ärzte und Therapeuten verwenden für ihre Entscheidungen zu Therapien mehr oder weniger bewusst Modelle, die sich sowohl von dem Modell des Patienten als auch von dem der Behandelnden untereinander unterscheiden können. So mag z.B. der Orthopäde die degenerativen Veränderungen, der Physiotherapeut die muskulären Dysbalancen und myofaszialen Triggerpunkte und der Psychologe Konflikte im häuslichen oder beruflichen Umfeld als Auslöser ansehen.

Um nicht nach dem Giesskannenprinzip jede Therapie über jeden Patienten $\mathrm{zu}$ "verschütten», sondern gezielt die therapeutischen Möglichkeiten sowohl der Schulmedizin als auch der Komplementärmedizin synergistisch einsetzen zu können, benötigen wir Ideen und Vorstellungen der Krankheiten und Wirkungsweisen der Therapien sowie daraus resultierende Konzepte.

Die in der Komplementärmedizin verwendeten Modelle des überlaufenden Fasses oder der Waage mit krankheitsfördernden/krankmachenden Faktoren auf der einen Seite und Ressourcen auf der anderen Seite spiegeln viele der oben beschriebenen «yellow

\section{KARGER}

Fax +497614520714 Information@Karger.com www.karger.com
(๑) 2013 S. Karger GmbH, Freiburg
Andreas Unger

Leitung Bereich Schmerztherapie, Aeskulap-Klinik

Gersauerstrasse 8, 6440 Brunnen, Schweiz

andreas.unger@aeskulap.com 
flags» im Sinne einer multikausalen Genese wider.

Mit der klassischen Differenzierung des Schmerzes in nozizeptiv, neuropathisch und «mixed pain» lassen sich Schmerzen zwar beschreiben und führen zu einem differenzierten Einsatz unterschiedlicher Substanzgruppen von Analgetika und Koanalgetika. Bei Therapieentscheidungen im Sinne einer kausalen Therapie helfen sie jedoch nicht weiter.

Bei der Betrachtung von Schmerzen im Bereich des Halte- und Bewegungsapparats mit der Vielzahl von Schmerzursachen und therapeutischen Möglichkeiten wird in unserer Klinik ein Modell der Ebenen verwendet (Abb. 1) - mit dem Ziel, Schmerzursachen $\mathrm{zu}$ differenzieren und daraus eine differenzierte kausale Therapie abzuleiten.

Eine der Ebene «Struktur» zuzuordnende Schmerzursache wie eine Schenkelhalsfraktur oder eine Hüftkopfnekrose ist weder mit noch so guter pharmakologischer Schmerztherapie noch mit Naturheilverfahren behandelbar und gehört in die Hände von Orthopäden/Unfallchirurgen. Auf der anderen Seite erklärt nicht jeder radiologisch nachgewiesene Befund auch die Schmerzen. Was die Frage aufwirft, wie vorzugehen ist, wenn der Bandscheibenvorfall links auftritt und die Schmerzen rechts sind.

Für eine gezielte Therapie sollten die im Vordergrund stehenden, aber auch die mit dem geringsten Aufwand und grössten Effekt behandelbaren Ursachen auf struktureller oder funktioneller Ebene herausgearbeitet werden.

Zunächst gilt es, die Schmerzen mittels einer umfangreichen Anamnese und einer ausführlichen funktionellen Untersuchung einer oder meist mehreren Ursachen bzw. Befunden zuzuordnen. Hintergrund dieser Diagnostik ist eine möglichst gezielte Therapie, die bei entsprechender Indikation auch einmal invasiv im Sinne einer Facetteninfiltration sein kann.

Wie bei jeder Erkrankung spielen natürlich allgemeine Risikofaktoren wie Ernährungsfehler, Bewegungs- mangel und psychosoziale Belastungen eine Rolle. So wird den meisten Patienten bei entsprechend plastischer Darstellung der Zusammenhang der lumbosakralen Schmerzen mit der abdominellen Adipositas, Hyperlordosierung und myostatischen Insuffizienz einleuchten. Mit dem Hinweis auf eine Lebensstiländerung wird sich der schmerzgeplagte Patient kaum zufriedengeben. Im Sinne einer ganzheitlichen Therapie sind diese Hinweise jedoch zu berücksichtigen, und die mögliche Wirkung von Heilfasten nicht nur auf den Metabolismus, sondern auch auf chronische Schmerzen sollte nicht unterschätzt werden.

In einer Kombination des Modells der Ebenen mit dem Fassmodell mit wellenförmiger (De-)Kompensationsschwelle lässt sich die Komplexität chronischer Schmerzen und deren Behandlung gut darstellen. Liegen alle vorhandenen Störungen und Belastungen - wie degenerative Veränderungen, biomechanische und myofasziale Funktionsstörungen oder Störfelder - noch unterhalb der Kompensationsschwelle, besteht Symptomfreiheit. Die Kompensationsschwelle schwankt abhängig von «Gesundheit», Infekten, Bewegungsmangel, Übergewicht, Essen, Trinken oder Rauchen (Abb. 2) und lässt sich durch Massnahmen wie Fasten, Entsäuern, Sauna, Kneipp, Qi Gong, Substitution von Vitaminen und Spurenelementen anheben. Dies hat zur Konsequenz, dass der Patient besser kompensieren kann (Abb. 3).

\section{Beispiel für eine Störung auf der Ebene der Struktur: Das Facettensyndrom}

Wenn die Symptomatik für ein mit starken strukturellen Veränderungen, d.h. einer Degeneration, verbundenes Facettensyndrom spricht, wird eine Serie Neuraltherapie meist nicht erfolgreich sein (Abb. 4), und eine durchleuchtungsgesteuerte Facetteninfiltration mit einem retardierten Kortikoid ist indiziert. Diese wird an unserer komplementärmedizinisch orientierten Einrichtung (AeskulapKlinik) mit einem C-Bogen ambulant oder stationär durchgeführt. Die Intervention dauert bei einem erfahrenen Schmerztherapeuten wenige Minuten, die Durchleuchtungszeit ist kürzer als eine Minute.

Wichtig ist, die Indikation aufgrund des klinischen Befunds und nicht aufgrund der radiologisch nachgewiesenen Spondylarthrose zu stellen, denn «infiltriert wird der Patient und nicht der Röntgenbefund».

Bestehen keine oder wenige andere Störungen, kann die Facetteninfiltration als alleinige therapeutische Methode zu einer Symptom- und Schmerzfreiheit (Kompensation) führen (Abb. 5).

\section{Beispiel für eine Störung auf der} Ebene der Funktionsstörung: Das myofasziale Triggerpunktsyndrom

Janet Travell hat bereits in den 1950erJahren das myofasziale Schmerzsyndrom beschrieben. Bei myofaszialen Triggerpunkten handelt es sich um muskuläre Dysfunktionen auf der Ebene der motorischen Endplatten und der sarkoplasmatischen Retikula, die zu lokalen Kontraktionen mit ischämieinduzierter Hypoxie führen. Wegweisend für die Diagnostik der myofaszialen Schmerzen ist die Kenntnis der für die einzelnen Muskeln typischen Übertragungsschmerzmuster («referred pain»), die von Travell und Simons [5] erfasst und in ihrem umfangreichen Werk sehr detailliert beschrieben werden.

Im Juli 2005 veröffentlichten Shah et al. [6] im Japanese Journal of Applied Physiology ihre Forschungsergebnisse, in denen sie in myofaszialen Triggerpunkten erhöhte Konzentrationen verschiedener Neuropeptide (Substanz P, Tumornekrosefaktor $\alpha$, Interleukin(IL)- $1 \beta$, IL-6 und IL-8, Calcitonin Gene-Related Peptide, Bradykinin, Serotonin und Norepinephrine) nachwiesen. Der pH-Wert des Gewebes war deutlich erniedrigt. 


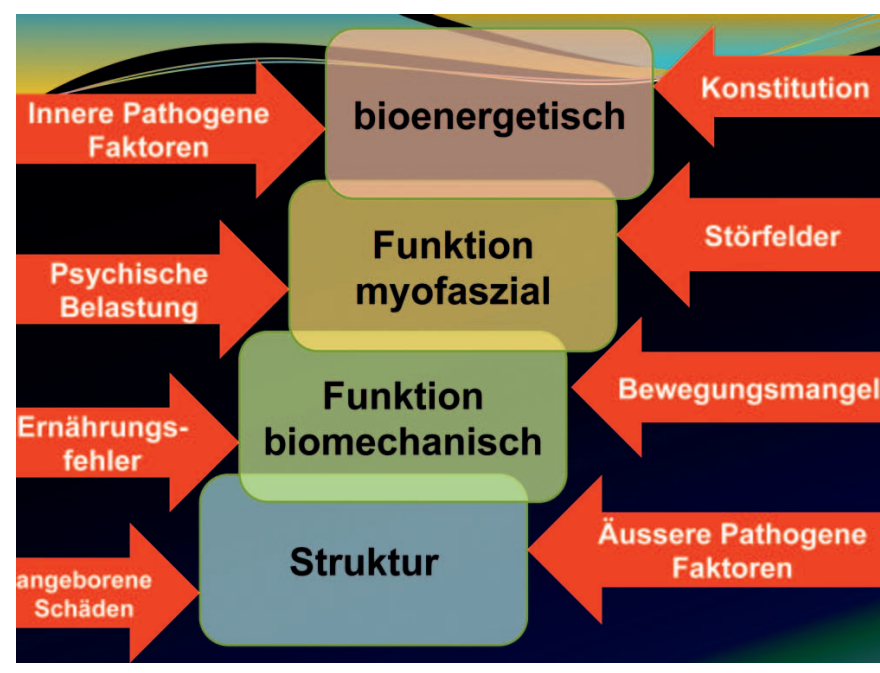

Abb. 1. Modell der Ebenen.

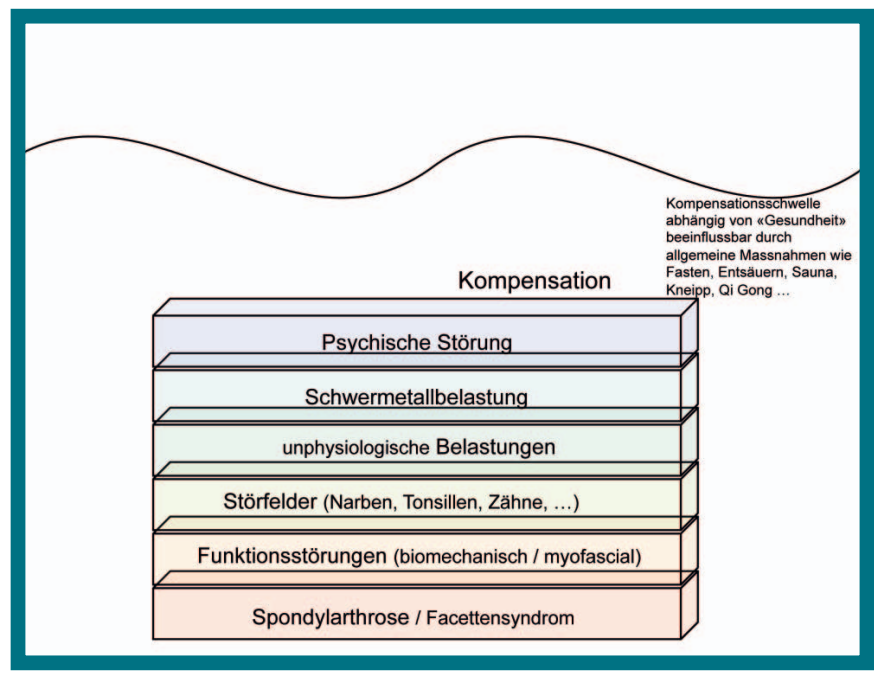

Abb. 3. Massnahmen wie Fasten, Entsäuern, Sauna, Kneipp und Qi Gong heben die Kompensationsschwelle an.

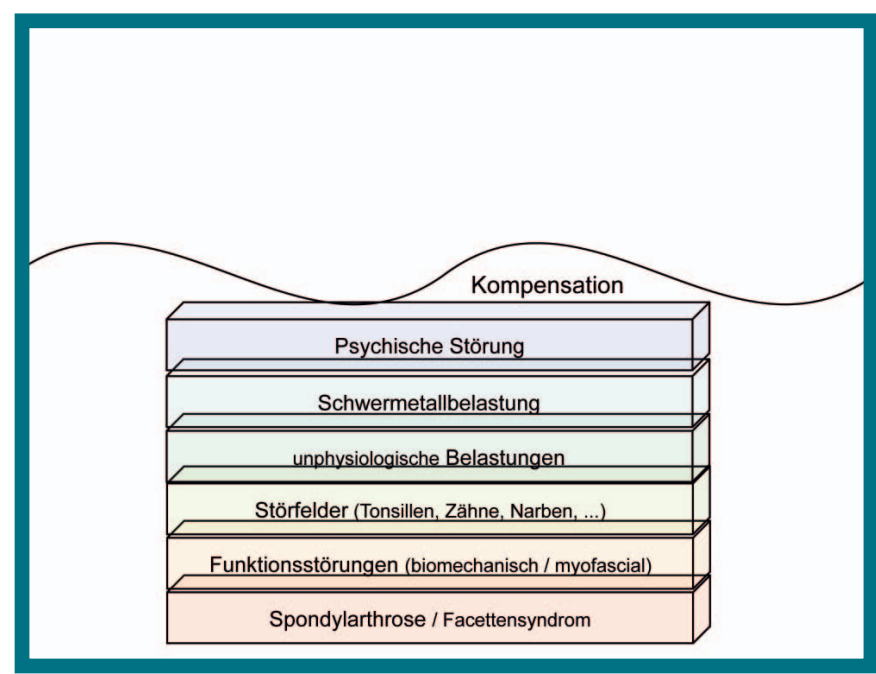

Abb. 5. Schmerzfreiheit nach Facetteninfiltration.

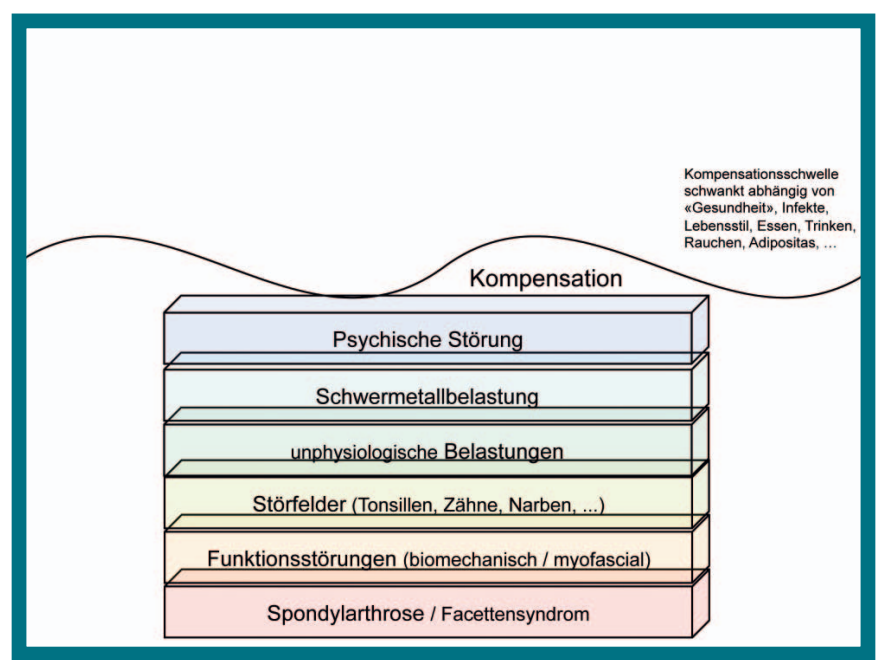

Abb. 2. Liegen alle Störungen und Belastungen unterhalb der Kompensationsschwelle, besteht Symptomfreiheit. Belastungen wie ein Infekt, Rauchen, Bewegungsmangel und Übergewicht senken die Schwelle.

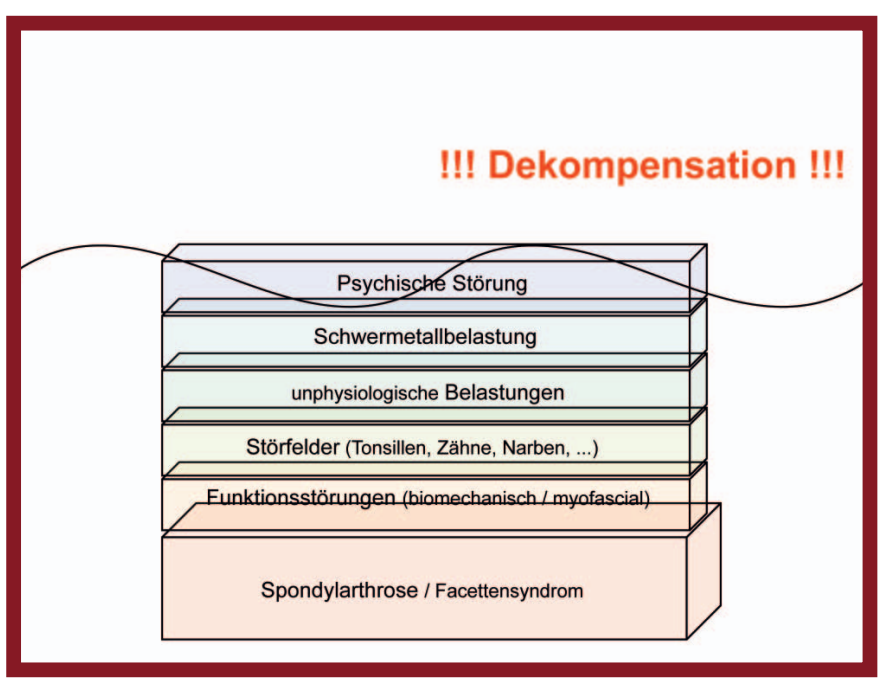

Abb. 4. Von einem Facettensyndrom ausgehende Schmerzen.

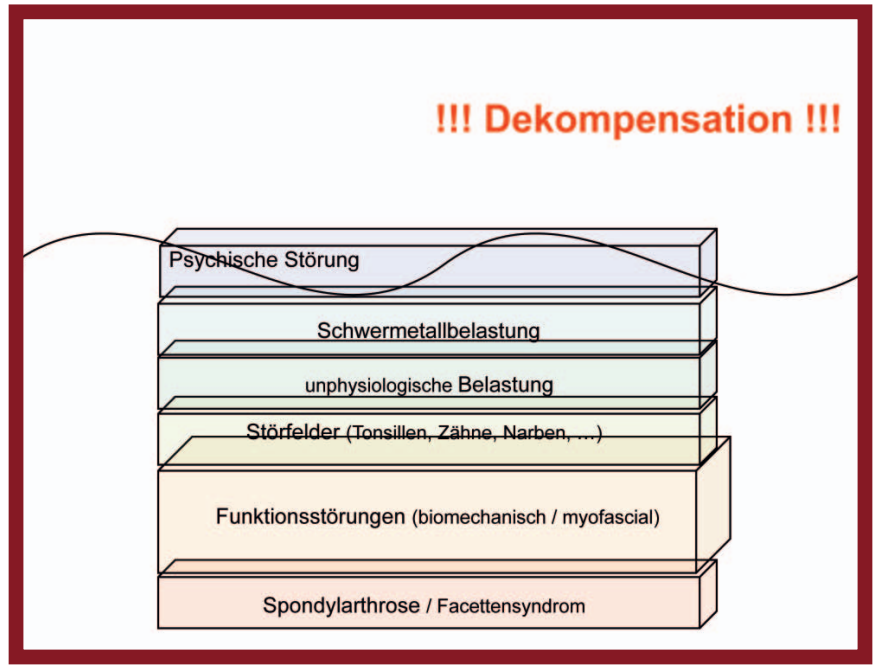

Abb. 6. Von einem myofaszialen Triggerpunktsyndrom ausgehende Schmerzen sind oft hartnäckig ... 
Nach der Behandlung der Triggerpunkte reduzierte sich die Konzentration dieser Entzündungsmediatoren drastisch und der $\mathrm{pH}$-Wert normalisierte sich wieder [6].

Neben dem von Travell und Simons beschriebenen «spray and stretch» und «needling» haben sich mittlerweile unzählige Methoden in der Behandlung myofaszialer Triggerpunkte etabliert. Zur Anwendung kommen verschiedene Infiltrations- und Akupunkturtechniken wie Körperakupunktur segmental (über die Muskelkette oder den Meridian) oder auch mit einer langen Nadel bis in den Triggerpunkt gestochen (dry needling), Somatopien (Ohrakupunktur, Yamamoto New System Acupuncture), osteopathische Verfahren wie Muskel-Energie-Techniken, StrainCounterstrain und myofasziales Release, Kinesiotaping, Feldenkrais-Methode usw.

Noch wenig verbreitet, aber hocheffektiv ist die Behandlung der myofaszialen Triggerpunkte mit extrakorporaler Stosswellentherapie (ESWT). Dabei wird sie sowohl als radiale (rESWT) als auch als fokussierte (fESWT) Massnahme eingesetzt. Die fESWT erscheint unserer Erfahrung nach in der Behandlung der myofaszialen Triggerpunkte deutlich überlegen.

So schmerzhaft und oft auch hartnäckig myofasziale Triggerpunktsyndrome sind (Abb. 6), so lassen sie sich doch meistens gut behandeln (Kompensation) (Abb. 7). Bei der Behandlung myofaszialer Triggerpunkte verschwimmt die Grenze zwischen schul- und komplementärmedizinischen Methoden.

\section{Beispiel für ein gemischt degeneratives und myofasziales Schmerzsyndrom}

Bei chronischen Rückenschmerzen finden sich meist mehrere Ursachen. Gänzlich ohne oder zumindest ohne anhaltenden Erfolg wird die Behand- lung myofaszialer Triggerpunkte bleiben, wenn zusätzlich ein von den kleinen Wirbelgelenken ausgehender starker nozizeptiver Schmerz im Sinne eines Facettensyndroms unbehandelt bleibt (Abb. 8, 9).

Ebenso würde in diesem Fall eine Facetteninfiltration nur einen Teil des Problems lösen und zu begrenzter Schmerzlinderung führen (Abb. 10). Allein eine kombinierte Behandlung führt hier zu einer Schmerzfreiheit (Kompensation) (Abb. 11). Facetteninfiltrationen führen in einem interdisziplinären Umfeld mit multimodalen Konzepten - mit Physiotherapie und psychologischer Begleittherapie durchgeführt - zu deutlich besseren Ergebnissen. Häufig handelt es sich nicht nur um eine Störung auf struktureller Ebene, sondern daneben auch auf myofaszialen und vielen anderen Ebenen. Wichtig ist es auch, den Patienten ganzheitlich mit seinen Störungen und Ressourcen wahrzunehmen.

Selbst invasive Verfahren wie die Facetteninfiltration werden frustran verlaufen, wenn der Patient von der Wirkungslosigkeit überzeugt ist (Nocebo-Effekt). Fortbestehende Schmerzen auch nach Ausschaltung der eigentlichen Schmerzursache beim chronifizierten Schmerz lassen sich durch die neuronale Plastizität (Schmerzgedächtnis) erklären. Auch darüber sollte mit dem Patienten gesprochen werden; «der Patient muss mitgenommen werden» (Abb. 12).

In diesem Zusammenhang kommt es schon auf die Wortwahl an. Patienten mit Schmerzen sind häufig davon überzeugt, eine schwere (unheilbare) Erkrankung zu haben. Was wird es wohl für Gefühle bei dem Patienten auslösen, wenn der Orthopäde das am Röntgenbildbetrachter hängende Desaster mit all den Osteochondrosen und Spondylophyten als Erklärung für die Schmerzen präsentiert (obwohl der möglicherweise Jahre ältere Nachbar bei weiter fortgeschrittenen degenerativen Veränderungen nie ernsthaft über Schmerzen klagt)?
Eine multikausale und komplexe Erkrankung bedarf einer ebenso vielschichtigen und komplexen Therapie

Nicht vernachlässigt werden darf bei chronischen Schmerzen die Behandlung von Störfeldern im Sinne der Neuraltherapie nach Huneke (Narben, Zahnherde usw.). Ein Fortbestehen von gravierenden Störfeldern kann alle Bemühungen wie Infiltrationen und Triggerpunktbehandlungen ins Leere laufen lassen (Abb. 13-15).

Neuraltherapie ist in der AeskulapKlinik eines der am häufigsten eingesetzten Verfahren. Thermoregulationsdiagnostik und Panoramaaufnahmen der Zähne gehören zu den Standardverfahren, die uns Hinweise auf Störfelder geben. Auch das Wissen um Meridianverläufe und segmentale Zusammenhänge helfen weiter. Neben der Störfeldbehandlung bietet die Neuraltherapie mit der segmentalen und erweiterten segmentalen Therapie über das vegetative Nervensystem weitere Möglichkeiten.

Häufig sehen wir den Einfluss einer Narbe nach Pfannenstielschnitt (suprapubisch) auf den lumbosakralen Übergang. Auch unerklärliche und unbeeinflussbare Halbseitenschmerzsyndrome lassen sich gelegentlich über diese Narbe behandeln und ein Sekundenphänomen nach Huneke auslösen. Selbstverständlich sollten auch die Narben nach Wirbelsäulenoperationen (Failed-back-surgery-Syndrom) behandelt werden. Als hocheffizient beobachten wir die Kombination von oberflächlichen mit tiefen Infiltrationen und anschliessenden osteopathischen Techniken sowie einer Atemtechnik, bei der die Patienten angeleitet in die Narbe «hineinatmen». Diese Kombinationen von verschiedenen Techniken können sehr tiefgreifende Wirkungen haben und sollten in einem ruhigen und für den Patienten sicheren Setting (gute Arzt-Patienten-Beziehung) durchgeführt werden, da starke psychische Reaktionen nicht selten sind. 


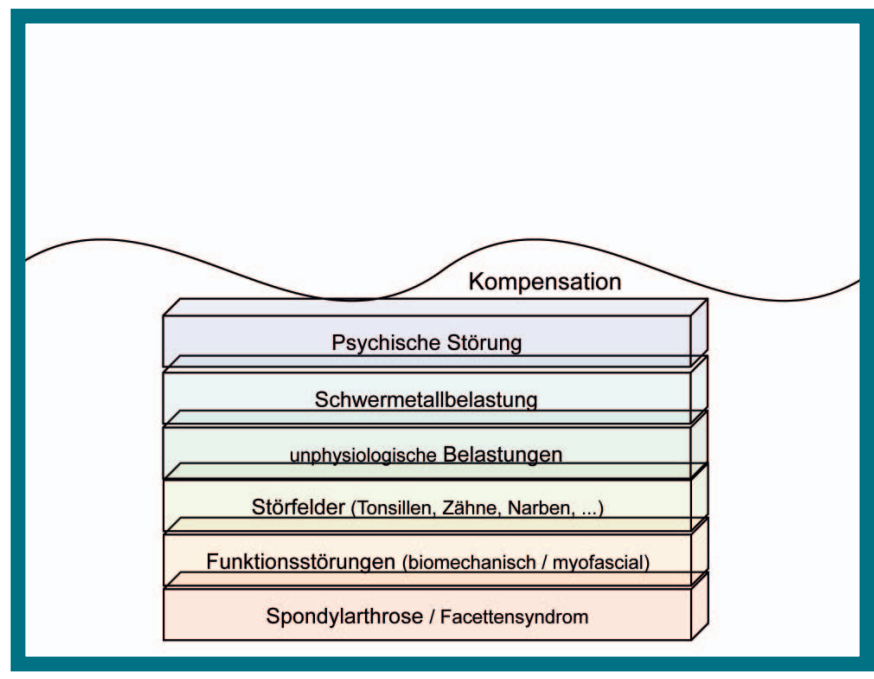

Abb. 7. ... lassen sich aber meistens gut behandeln (Kompensation).

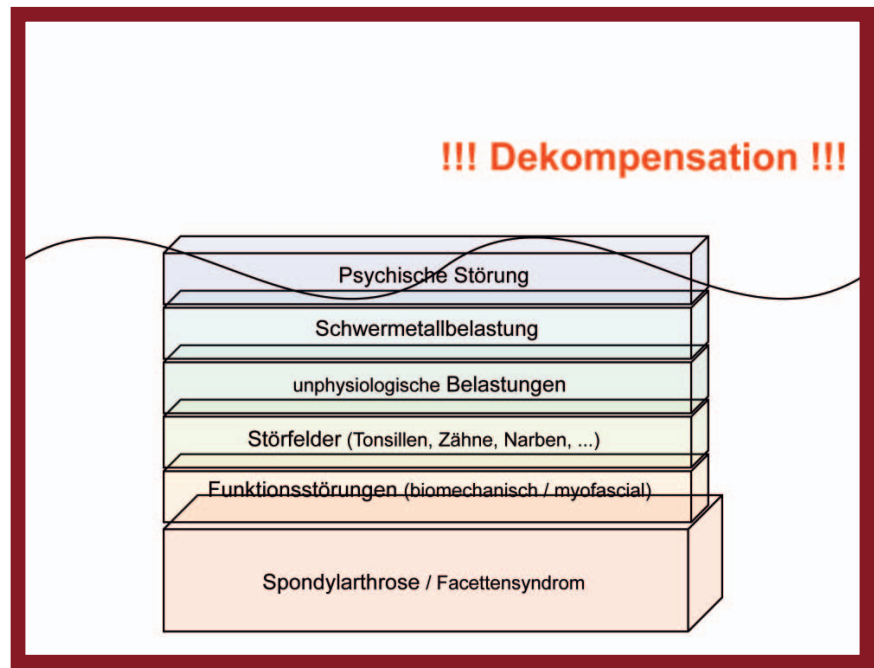

Abb. 9. Würde man in diesem Fall nur die myofaszialen Triggerpunkte behandeln, würde eine Besserung, aber nicht Schmerzfreiheit erreicht werden.

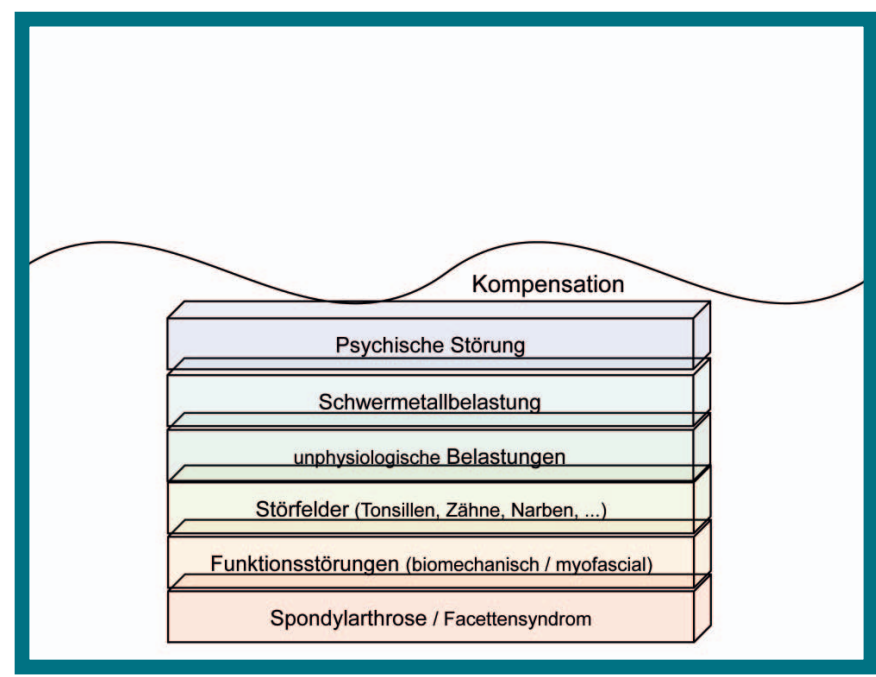

Abb. 11. Allein mit einer Kombination der Therapien auf den Ebenen der Struktur und der Funktionsstörung ist Schmerzfreiheit erreichbar.

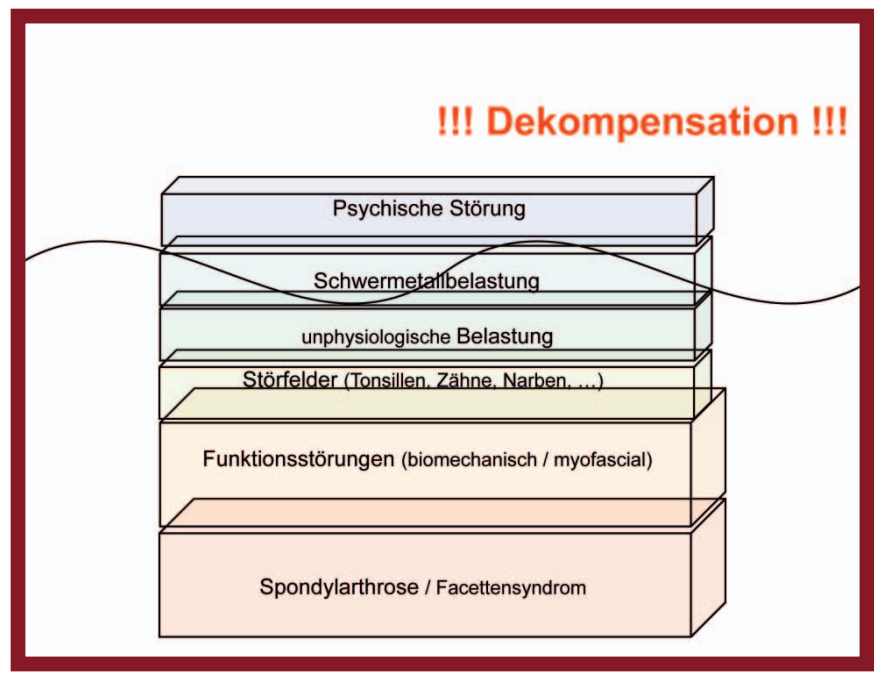

Abb. 8. Meistens finden sich bei chronischen Rückenschmerzen mehrere Ursachen, die auch einer Behandlung bedürfen.

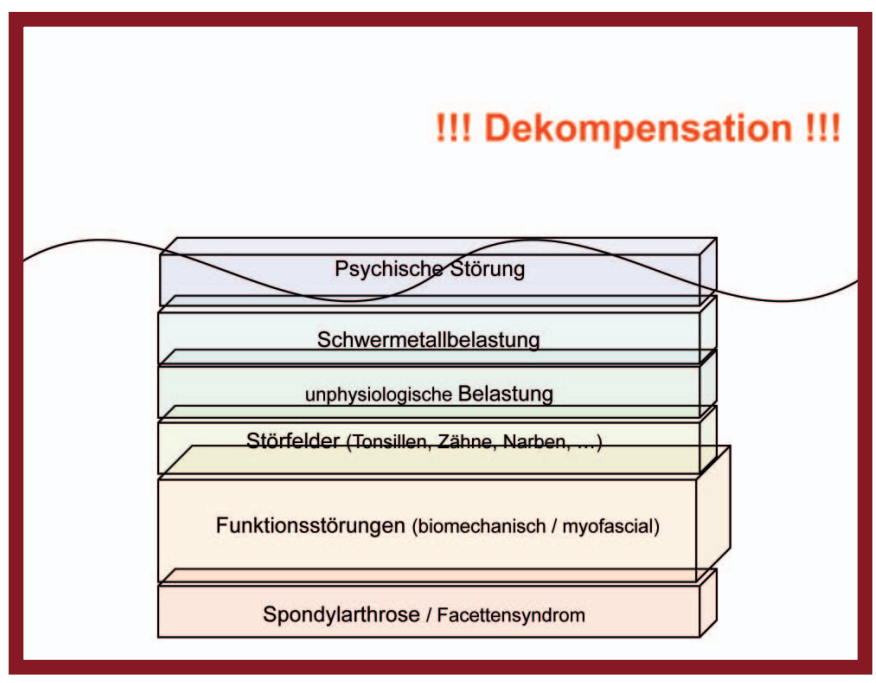

Abb. 10. Ebenso wenig wäre allein mit einer Facetteninfiltration eine Schmerzfreiheit zu erreichen.

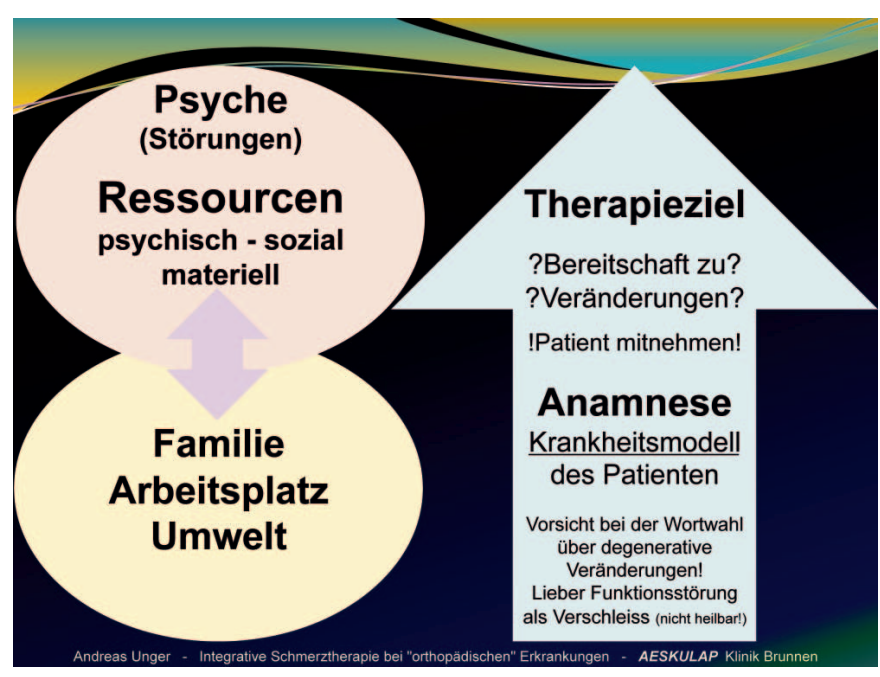

Abb. 12. Der Patient muss in seiner Komplexität wahrgenommen und «mitgenommen» werden. 


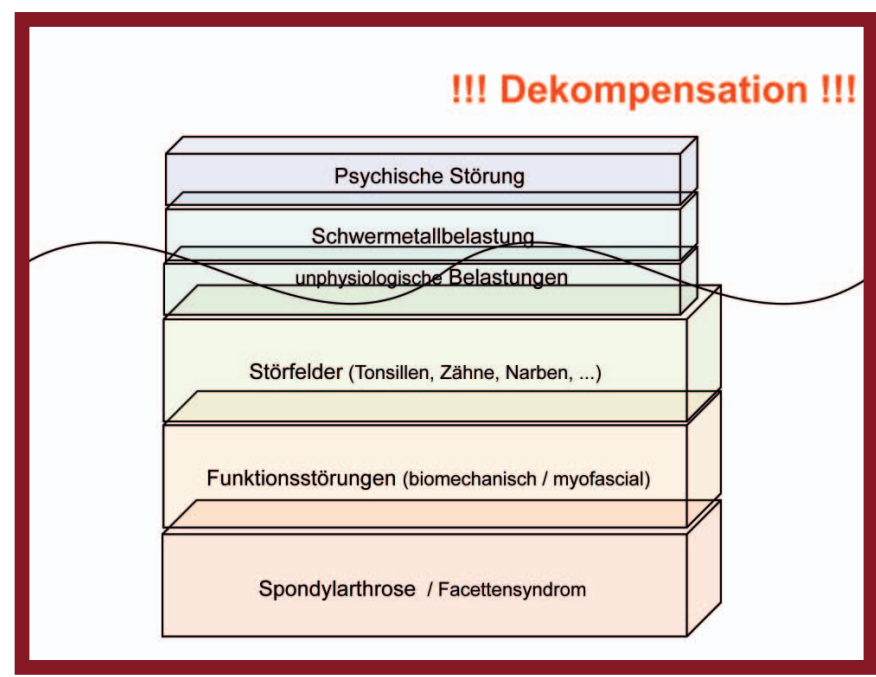

Abb. 13. Häufig finden sich zusätzlich Störfelder und andere Belastungen.

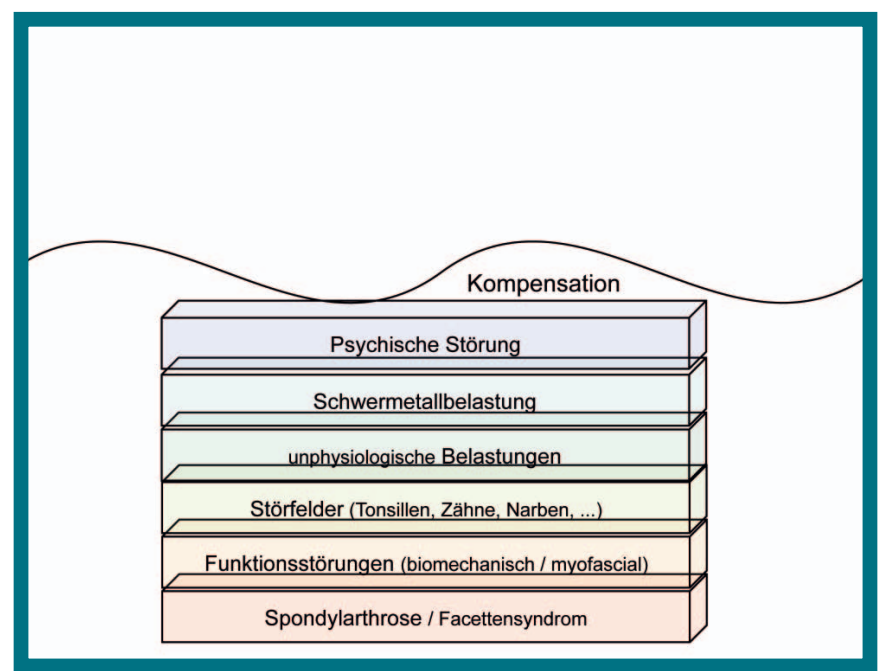

Abb. 15. Bei der Therapie des chronischen Rückenschmerzes ist die Neuraltherapie ein wichtiger Baustein.

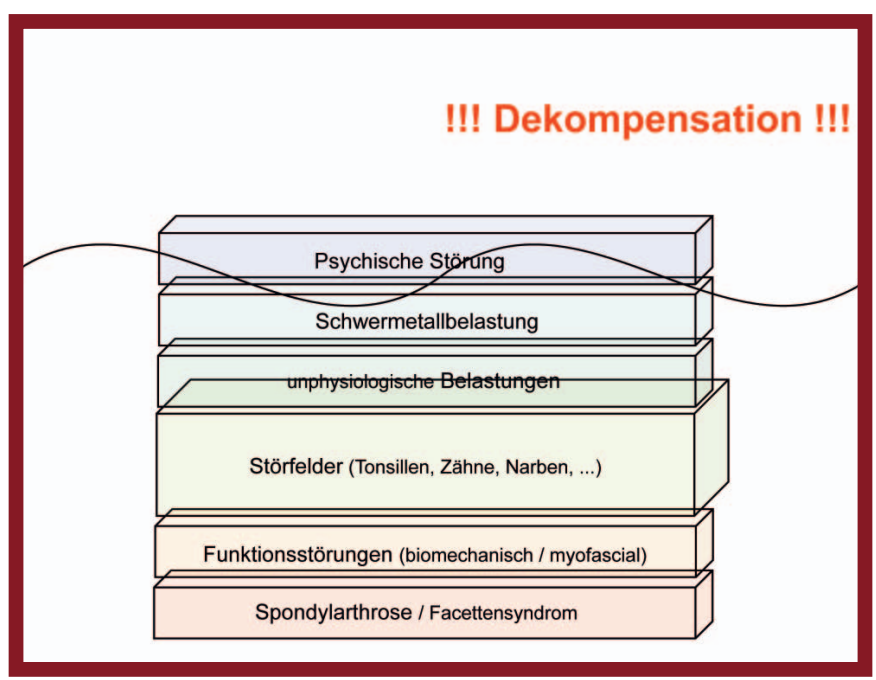

Abb. 14. Eine Vernachlässigung der Störfelder wird zu unbefriedigenden Ergebnissen führen.

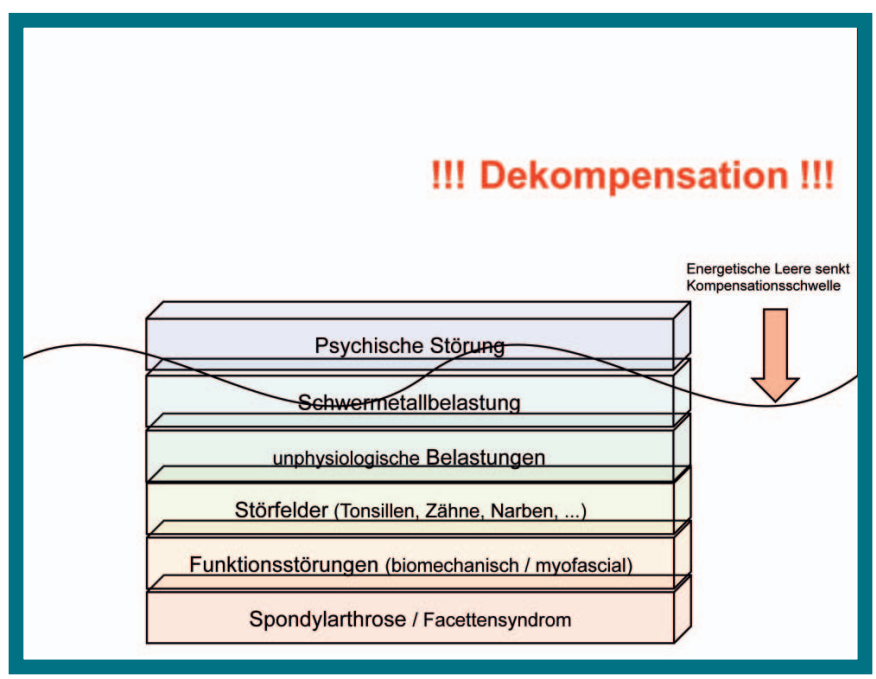

Abb. 16. Energetische Leere.

\section{Literatur}

Eine therapeutische Herausforderung stellen Patienten in einem energetischen Leerezustand (Abb. 16) oder einer Reaktionsstarre dar.

\section{Fazit}

Die Komplexität der Krankheitsursachen liesse sich problemlos noch um Einiges steigern. Dennoch wird bereits deutlich, dass die Schmerztherapie von chronischen Rückenschmerzen, wenn wir sie als kausale Therapie und nicht als ein «Verwalten von Schmerzen» mit (hochdosierten) Analgetika verste- hen, ebenso vielschichtig und komplex sein muss, um dem Schmerz gerecht zu werden. Das erfordert in verschiedenen Verfahren erfahrene und engagierte Ärzte und Therapeuten und erklärt auch, warum Schmerztherapie ein Team benötigt und keine "OneMan-Show» sein kann.

In der Schmerztherapie verschwimmen die Grenzen zwischen schul- und komplementärmedizinischen Methoden. Integrative Medizin ist keine Frage des Glaubens oder der Einstellung, sondern bei der Komplexität der Schmerzerkrankungen schlichtweg eine Notwendigkeit.
1 Schmidt CO, Raspe H, Pfingsten M, Hasenbring M, Basler HD, Eich W, Kohlmann T: Back pain in the German adult population: prevalence, severity, and sociodemographic correlates in a multiregional survey. Spine 2007;32:2005-2011.

2 Nationale VersorgungsLeitlinie Kreuzschmerz. Kurzfassung. Version 1.2, August 2011. AWMF-Register Nr. nvl/007.

3 Manek NJ, Nisha J, MacGregor AJ: Epidemiology of back disorders: prevalence, risk factors, and prognosis. Curr Opin Rheumatol 2005; 17:134-140.

4 Weinschenk S (Hrsg): Handbuch der Neuraltherapie. München, Elsevier/Urban \& Fischer, 2010.

5 Travell JG, Simons DG: Myofascial Pain and Dysfunction. Philadelphia, Lippincott Williams \& Wilkins, 1983.

6 Shah JP, Philipps TM, Danoff JV, Gerber LH: An in vivo microanalytical technique for measuring the local biochemical milieu of human skeletal muscle. J Appl Physiol 2005;99:1977-1984. 